\title{
Quantum Entropic Gravity with Quantum Field Theory and General Relativity : A First Approach with Bijective Physics
}

Roman Baudrimont

Bijective Physics Institute

RomanBaudrimont.cd@gmail.com

\begin{abstract}
The purpose of this paper is to find a quantum formulation of entropic gravity, incorporating the bijective theory. In a first part, we will describe the basis of the bijective theory. In a second part, we will incorporate the stress energy quantum tensor defined in the linear approximation of the Einstein's equation in its quantum version to entropy. In the third part, we will integrate the bijective theory with the newly created relativistic quantum entropy. This will lead us four part where we will formalize quantum entropic gravity.
\end{abstract}

Keywords: Entropic Gravity, Bijective Physics, Quantum Field Theory, Stress Energy Tensor

\section{Part 1 - The Bijective Theory}

The bijective theory ${ }^{[1-2]}$ is a theory that is born from the principle of the epistemological stability of a theory. To know wheter a theory is " stable » epistemologically, we define physical reality by bijective function of set theory.

Thus, this bijective function prescribes for every element of know reality exactly as element of the model. In other words, each element of the model corresponds exactly to an element in physical reality, so there is a bijective correspondance between the elements of the model and the physical reality.

For example, the elements of $X$ are in bijective correspondence with the elements of $Y$, so to have $X(1) \rightarrow$ $Y(D)$, with the bijective function $f(1)=D$, puis $X(2) \rightarrow Y(B)$, with the bijective function $f(2)=B$ etc.

Based on NASA's discovery that universal space has a euclidian form and based on the fact that the Planck's units are possible properties of universal space, the bijective theory defines the " Euclidian-Planck Metrics " (EMP) wherein each volume of Planck contains Planck energy, which means that the empty universal space of field energy can by defined by Planck energy density, we will note :

$$
\rho_{P E}=\frac{E_{p}}{l_{p}^{3}}=\frac{c^{7}}{h g^{2}}
$$

Each elemental particle without inertial mass will change the Euclidean Planck Metrics, decreasing the energy density of the Planck space proportionally to its energy $E$, so we have:

$$
\rho_{P E}=\frac{E_{p}}{l_{p}^{3}}=\frac{c^{7}}{h g^{2}}
$$

We can have, finally :

$$
\rho_{q v E}=\rho_{P E}-\frac{m c^{2}}{V}
$$

Where $\rho_{q v E}$ is the density energy of quantum vacuum in the center of particle (or massive body). We can get the mass energy equivalence, so $E=m c^{2}=\Delta_{E P M} \cdot V$ with $\Delta_{E P M}=\rho_{q v E}-\rho_{P E}$. 
From the equivalence principle of general relativity, we can have :

$$
m_{P i}=m_{P g}=\frac{\Delta_{E P M} \cdot V}{c^{2}}
$$

The "ether" is the "dynamic quantum vacuum", where he is given a physical property of planck energy density, which has minimal variations according to the mass a given physical object : diminished energy density of ether corresponds to the mass of a given physical object. So, this equation is a bijective physics, where the diminished energy density is a bijective correspondence with mass of physical object.

In special relativity, we can have $E_{\text {special }}=\gamma m c^{2}=\gamma \cdot \Delta_{E P M} \cdot V$, with $\gamma$ is equal to, in bijective physics :

$$
\gamma=\frac{1}{\sqrt{\frac{v^{2}}{c^{2}}}}=\frac{m c^{2}}{\Delta_{E P M} \cdot V}
$$

So, in the special relativity, using (5), the relativist rate of clock is :

$$
\Delta t_{0}=\Delta t \frac{m c^{2}}{\Delta_{E P M} \cdot V}
$$

The time depend only on the variability of $\Delta_{E P M}$, the energy $m c^{2}$ and volume $V$. The time not depend to the position of the observer. The bijective physics give a new interpretation on the special relativity.

\section{Part 2 - Entropic formulation of the stress energy quantum tensor.}

\section{Part A - First formulation based on the linear approximation of the Einstein's Equation.}

A first mathematical equality consists in writing that entropy is proportional to pressure and volume. By writing $p$ the pressure and $V$ a volume, we can determine the entropy $S$ :

$$
S T=P V
$$

With $T$ the temperature in kelvin of the studied system. If we consider a Riemanian space ${ }^{[3]}$, we can define the volume by the different coordinates $u^{i}$ and the metric tensor $g_{i j}$, so we have:

$$
V=\int \sqrt{|g|} \sum_{i=1}^{n} d u^{i}
$$

Considering that the stress energy tensor is a stress tensor ${ }^{[4]}$, we can use the linear approximation of the Einstein's equation to write the following equality, with $\Gamma(r, t)$ the scalar form of the stress energy tensor in general relativity ${ }^{[5]}$ :

$$
P=\Gamma(r, t)
$$

Using equality (7), (8) and (9) we have, finally, the following equality :

$$
S T=\Gamma(\vec{r}, t) \int \sqrt{|g|} \sum_{i=1}^{n} d u^{i}
$$


By isolating the entropy, we get :

$$
S(\vec{r}, t)=\Gamma(\vec{r}, t) \frac{1}{T} \int \sqrt{|g|} \sum_{i=1}^{n} d u^{i}
$$

Entropy becomes a scalar, geometrically dependent on space, since it depends on the metric tensor of coordinates $d u^{i}$. But entropy is also dependant on the temperature (in Kelvin) and, most importantly, on the pressure or the energy density.

We can say that the higher the pressure, the stronger the entropy. In the thermodynamics of the Black Holes (which we will not stydy here), the pressure exerted by these massive bodies is gigantic, and their entropy is extremely strong.

\section{Part B - Second formulation based on the Klein-Gordon Equation}

The Klein-Gordon equation is ${ }^{[6]}$ :

$$
\left(\Delta-\frac{1}{c^{2}} \frac{\partial^{2}}{\partial t^{2}}\right) \psi(\vec{r}, t)=\frac{m^{2} c^{2}}{h^{2}} \psi(\vec{r}, t)
$$

And the linear approximation of Einstein's equation is ${ }^{[5]}$ :

$$
\left(\Delta-\frac{1}{c^{2}} \frac{\partial^{2}}{\partial t^{2}}\right) h(\vec{r}, t)=\frac{8 \pi G}{c^{4}} \Gamma(\vec{r}, t)
$$

We have with (12) and (13) the following equality :

$$
\Gamma(\vec{r}, t)=K^{-1} \frac{c^{2} m^{2}}{h^{2}} \psi(\vec{r}, t)
$$

We can nest it in our simple formulation of entropy that we saw in our first formulation, which allows to obtain the following equation :

$$
S(\vec{r}, t)=\frac{1}{T}\left[K^{-1} \frac{c^{2} m^{2}}{h^{2}} \int \sqrt{|g|} \sum_{i=1}^{n} d u^{i}\right] \psi(\vec{r}, t)
$$

The terms between hook correspond to energy. It is easy to see that the entropy thus defined is similar to the entropy of Clausius, which is expressed as follows :

$$
S=\frac{E}{T}
$$

Except that the entropy of clausius becomes probabilistic, since we have the complex function $\psi(\vec{r}, t)$, which gives :

$$
S(\vec{r}, t)=\frac{E}{T} \psi(\vec{r}, t)
$$

It is easy to see that if we make the norm squared of the following equation, we fall back on the entropy of Clausius that we know well. Now we can transform the wave function $\psi(\vec{r}, t)$ by the complex probabilistic function with vector values :

$$
S(\vec{r}, t)=\frac{1}{T}\left[K^{-1} \frac{c^{2} m^{2}}{h^{2}} \int \sqrt{|g|} \sum_{i=1}^{n} d u^{i}\right]|\psi(\vec{r}, t)\rangle
$$




\section{Part 3 - Quantum entropy in bijective physics}

\section{Part A - Calculation of entropic gravity in bijective physics}

As we said above, we can consider $\Gamma(\vec{r}, t)$ as a pressure but also as a density of energy. Therefore, by relying on the bijective theory ${ }^{[1-2]}$, we can write these equations:

$$
\begin{gathered}
\Gamma_{P E}(\vec{r}, t)=\rho_{P E} \\
\Gamma_{Q V}(\vec{r}, t)=\rho_{q v E} \\
\Gamma_{E P M}(\vec{r}, t)=\Gamma_{P E}(\vec{r}, t)-\Gamma_{Q V}(\vec{r}, t)
\end{gathered}
$$

With this following equation (19), (20) and (21), we can write the stress energy quantum tensor by the bijective physics :

$$
\begin{gathered}
\Gamma_{P E}(\vec{r}, t)-\Gamma_{q v E}(\vec{r}, t)=K^{-1} \frac{c^{2} m^{2}}{h^{2}} \psi_{E}(\vec{r}, t) \\
\Gamma_{E P M}(\vec{r}, t)=K^{-1} \frac{c^{2} m^{2}}{h^{2}} \psi_{E P M}(\vec{r}, t)
\end{gathered}
$$

By taking the following equality, we can nest it in our simple formulation of entropy that we saw in our first formulation, which allows to obtain the following equation :

$$
\begin{gathered}
\mathrm{S}_{E P M}(\vec{r}, t)=\left(\Gamma_{P E}(\vec{r}, t)-\Gamma_{q v E}(\vec{r}, t)\right) \frac{1}{T} \int \sqrt{|g|} \sum_{i=1}^{n} d u^{i} \\
\mathrm{~S}_{E P M}(\vec{r}, t)=\Gamma_{E P M}(\vec{r}, t) \frac{1}{T} \int \sqrt{|g|} \sum_{i=1}^{n} d u^{i}
\end{gathered}
$$

So, by breaking down the stress energy quantum tensor, we have :

$$
\mathrm{S}_{E P M}(\vec{r}, t)=\frac{1}{T}\left[K^{-1} \frac{c^{2} m^{2}}{\hbar^{2}} \int \sqrt{|g|} \sum_{i=1}^{n} d u^{i}\right] \psi_{E P M}(\vec{r}, t)
$$

We fall back on the same formula of entropy as that obtained previously.

Thus, according to bijective methodology, the entropy is proportional to the wave function and the energy density itself can be described from the geometry of the space and its energy.

\section{Part A - Calculation of entropic gravity in bijective physics}

We can rapproach the Entropic Gravity in the equation of the energy equation of bijective physics ${ }^{[1-2]}$, who the formulation is :

$$
E=m c^{2}=\left(\rho_{P E}-\rho_{q v E}\right) \cdot V
$$

We have :

$$
T \cdot \mathrm{S}_{E P M}(\vec{r}, t)=\left(\Gamma_{P E}(\vec{r}, t)-\Gamma_{q v E}(\vec{r}, t)\right) \cdot \int \sqrt{|g|} \sum_{i=1}^{n} d u^{i}
$$


Where $T \cdot \mathrm{S}_{E P M}(\vec{r}, t)=E=m c^{2}$, then $\Gamma_{P E}(\vec{r}, t)-\Gamma_{q v E}(\vec{r}, t)=\left(\rho_{P E}-\rho_{q v E}\right)$ and $\int \sqrt{|g|} \sum_{i=1}^{n} d u^{i}$ is the same of the volume $V$. We have finally :

$$
T \cdot \mathrm{S}_{E P M}(\vec{r}, t)=\left[K^{-1} \frac{c^{2} m^{2}}{h^{2}} \int \sqrt{|g|} \sum_{i=1}^{n} d u^{i}\right] \psi_{E P M}(\vec{r}, t)
$$

Were $K^{-1} \frac{c^{2} m^{2}}{h^{2}}$ is the energy density and $\int \sqrt{|g|} \sum_{i=1}^{n} d u^{i}$ the volume.

\section{Part 4 - Quantum entropic gravitation and bijective physics}

According to the Verlinde's equation of the entropic gravity ${ }^{[7]}$, who is :

$$
T \frac{\partial S}{\partial X}=G \frac{m M}{R^{2}}=\frac{N K_{b} T}{X}
$$

We can have the following equation :

$$
T \frac{\partial S}{\partial X}=\frac{\partial}{\partial X}\left\{\left[K^{-1} \frac{c^{2} m^{2}}{h^{2}} \int \sqrt{|g|} \sum_{i=1}^{n} d u^{i}\right] \psi(\vec{r}, t)\right\}=G \frac{m M}{R^{2}}
$$

The following equation no depends of the temperature in kelvin, depend on the energy and geometry of the space and finally the wave quantum function.

More precisely, quantum entropic gravity depends exclusively on mass, volume and wave function.

Following the equation (24) and (25), we have quantum entropic gravitation in bijective physics :

$$
\begin{gathered}
T \frac{\partial \mathrm{S}_{E P M}(\vec{r}, t)}{\partial X}=\frac{\partial}{\partial X}\left\{\left(\Gamma_{P E}(\vec{r}, t)-\Gamma_{q v E}(\vec{r}, t)\right) \frac{1}{T} \int \sqrt{|g|} \sum_{i=1}^{n} d u^{i}\right\}=G \frac{m M}{R^{2}} \\
T \frac{\partial \mathrm{S}_{E P M}(\vec{r}, t)}{\partial X}=\frac{\partial}{\partial X}\left\{\Gamma_{E P M}(\vec{r}, t) \frac{1}{T} \int \sqrt{|g|} \sum_{i=1}^{n} d u^{i}\right\}=G \frac{m M}{R^{2}}
\end{gathered}
$$

So, quantum entropic gravity is a bijective physics, he is in a bijective correspondance with the EuclidianPlanck Metrics. Quantum entropic gravity depends only on the density energy and on the structural form of space.

\section{Part 5 - Conclusion.}

Quantum entropic gravity, deduced from the quantum field theory and the linear approximation of Einstein's equation, allows to get a quantum entropic gravity with with a independant time, and entirely depenent on energy density and the geometric space. Entropic quantum gravity, analysed with the bijective theory, confirms its hypotheses.

Therefore, the quantum entropic gravity respects the bijective physics : he is in a bijective correspondance with the Euclidian-Planck Metrics, where the diminished energy density is a bijective correspondence with mass of physical object. Quantum entropic gravity allows to describe the physic reality. 
Part 6 - Bibliography.

[1] Amrit Sorli (2018), Bijective Analysis of Physical Equations and Physical Models, NeuroQuantology, Volume 16, issue 7, DOI: 10.14704/nq.2018.16.7.11684

[2] Amrit Sorli, Uros Dobnikar, Santanu Kumar Patro, Magi Mageshwaran, David Fiscaletti (2018), Euclidian-Planck Metrics of Space, Particle Physics in Cosmology, NeuroQuantology, Volume 16, issue 4, DOI: 10.14704/nq.2018.16.4.1221

[3] Elie Cartan (1925), La géométrie des espaces de Riemann, Mémorial des sciences mathématiques, fascicule 9, p1-61

[4] Eric Gourgoulhon (2013-2014), Relativité Générale, Observatoire de Paris, Master Astronomie Astrophysique et Ingénierie Spatiale année M2 parcours de recherche, Ondes gravitationnelles page 138-145

[5] Yurij V. Baryshev (2017), Fondation of relativistic astrophysics : Curvature of Riemannian Space versus Relativistic Quantum Field in Minkowski Space, Arxiv 1702.02020v1, page 17, $18,28-30$

[6] J.F. Fortin, P.O. Genest, J.F. Laprise, S. Marchand, V. Bérubé et S. Bégin (2003), Les outils de base de la physique des particules, Luc Marleau, université LAVAL

[7] Eric Verlinde (2010), On the Origin of Gravity and the Laws of Newton, arXiv:1001.0785v1 